\title{
LES PRODUCTIONS AUDIOVISUELLES DES JOUEURS DE JEUX VIDÉO
}

\author{
Entre formation professionnelle et apprentissages esthétiques autodidactes \\ Nicolas Auray et Fanny Georges
}

\section{La Découverte I Réseaux}

\author{
$2012 / 5-n^{\circ} 175$ \\ pages 145 à 173
}

ISSN 0751-7971

Article disponible en ligne à l'adresse:

http://www.cairn.info/revue-reseaux-2012-5-page-145.htm

Pour citer cet article :

Auray Nicolas et Georges Fanny, «Les productions audiovisuelles des joueurs de jeux vidéo » Entre formation professionnelle et apprentissages esthétiques autodidactes,

Réseaux, 2012/5 n¹75, p. 145-173. DOI : 10.3917/res.175.0145

Distribution électronique Cairn.info pour La Découverte.

(C) La Découverte. Tous droits réservés pour tous pays.

La reproduction ou représentation de cet article, notamment par photocopie, n'est autorisée que dans les limites des conditions générales d'utilisation du site ou, le cas échéant, des conditions générales de la licence souscrite par votre établissement. Toute autre reproduction ou représentation, en tout ou partie, sous quelque forme et de quelque manière que ce soit, est interdite sauf accord préalable et écrit de l'éditeur, en dehors des cas prévus par la législation en vigueur en France. II est précisé que son stockage dans une base de données est également interdit. 


\title{
LES PRODUCTIONS AUDIOVISUELLES DES JOUEURS DE JEUX VIDÉO :
}

\author{
Entre formation professionnelle \\ et apprentissages esthétiques autodidactes
}

Nicolas AURAY Fanny GEORGES 
a numérisation culturelle a suscité deux effets contradictoires. D'une part, le numérique a modifié les circuits de consécration autour des œuvres, car il va de pair avec un affaiblissement de la critique professionnelle et experte, laquelle se trouve fragilisée par l'avènement des modalités de recommandation horizontales, et par de nouveaux circuits de prescription orientés autour de la construction d'une audience sur le Web. Ce remplacement partiel de la critique experte est particulièrement ressenti dans un contexte où la diversification des formats et des genres et l'hétérogénéité des créations rendent plus flous les « cadres d'interprétation » par lesquels sont structurés les attentes de qualité et les schémas de catégorisation des récepteurs. D'autre part, le numérique a favorisé l'abaissement des barrières à l'entrée pour produire et éditer, en donnant une nouvelle chance aux autodidactes et aux francs-tireurs. Le nouveau média qu'est Internet s'est ainsi montré susceptible de permettre à des amateurs, " enrichis » par leurs apprentissages dans des communautés de pratique (Beuscart et al., 2009), de voir leurs productions rivaliser avec celles de professionnels, comme c'est le cas dans différents domaines audiovisuels - photographie (Gunthert, 2008), court-métrage (Bourgatte et Thabourey, 2012), musique.

Le jeu vidéo est placé au cœur de cette double transformation. Du fait que son développement a coïncidé avec l'émergence du réseau, il a vu la critique horizontale et les recommandations dominer ses formes de légitimation. En même temps, le jeu vidéo présente l'originalité d'être un divertissement qui a beaucoup mis en avant la créativité ${ }^{1}$ des utilisateurs, leur capacité à marquer un écart ou une différence avec le programme qu'on cherche à leur imposer (Silverstone, 1989), et accessoirement la possibilité de partager et cumuler les traces de leurs performances d'usage ou de leurs détournements. L'univers des

1. La reconnaissance de la créativité des utilisateurs avait été ouverte par un changement de paradigme des études de la réception : le courant des "Uses and gratifications 》 a cherché à s'extraire du «médiacentrisme » en transformant le sens de la question posée par les fondateurs des études de communication. Ils ne demandent plus comment les médias influencent les personnes, mais ce que font les gens avec les médias. Ce changement de paradigme va ouvrir l'espace de recherche à une autre conception de la relation des usagers avec les produits de l'industrie culturelle. Sur un tel changement de paradigme, cf. Maigret (2000). 
" game studies » est abondant en éloges des formes de narration « ouvertes " (Aarseth, 2001 ; Costikyan, 2002) et en description des multiples formes de modifications et de subversion des produits par les utilisateurs (Taylor, 2006). L'approche par les «nouveaux médias » a quant à elle insisté sur les expressions de ces contenus dans des formes esthétiques comme des clips vidéo, des bandes dessinées, des films ou des musiques (Manovitch, 2007 ; Sihvonen, 2009). Dans un tel contexte, les créations faites à partir de jeux vidéo sont un exemple de jouabilité émergente (" emergent gameplay»), terme regroupant les utilisations nouvelles et inattendues d'un jeu vidéo comme sa modification notamment à des fins artistiques. Elles font partie des " remédiations " utilisant le moteur de jeu pour la création d'artefacts externes tels que des collections d'images en ligne fondées sur des copies d'écran, des romansphotos, des films faits à partir de la capture de données en temps réel durant les sessions de jeux, et légèrement retouchés et montés ultérieurement (Bolter et Grusin, 2000).

Le jeu vidéo occupe en conséquence une place centrale dans l'apparition d'audiences actives, c'est-à-dire dans l'émergence de pratiques créatives dans la réception des industries culturelles, jusqu'alors sérielle et marquée par l'existence d'audiences passives. Mais, en outre, il constitue un observatoire privilégié pour étudier des dynamiques d'apprentissages alternatives aux circuits de formation professionnelle. Ces « dynamiques d'apprentissage » alternatives renvoient à deux modalités complémentaires d'acquisition des compétences : elles désignent d'une part les mécanismes d'inculcation d'un " goût cultivé » par l'intériorisation des schèmes de différenciations internes entre les styles de jeux vidéo ; elles désignent d'autre part la formation à la grammaire cinématographique permettant au jeu vidéo de constituer une expérience cohérente. Ces deux dynamiques d'apprentissage, dans le cas du jeu vidéo, donnent un rôle clef aux voies autodidactes et à l'insertion dans des communautés amateurs. Du fait de l'importance de la critique amateur et des recommandations communautaires venues de joueurs, les mécanismes de construction du " goût », c'est-à-dire de hiérarchisation entre les genres et, à l'intérieur des genres, de hiérarchisation entre les œuvres, se sont en grande partie effectués autour de communautés de joueurs échangeant des vidéos filmant des extraits de sessions de jeu et échangeant en voix off leurs avis. Ces communautés de testeurs se sont animées et structurées sur Internet. Le défaut de «patrimonialisation » de l'industrie du jeu vidéo a ainsi été compensé par l'essor de circuits de reconnaissance éclairée et de formes curatoriales sur le réseau (Balzerani, 2009). Parallèlement, la facilité qu'ont les utilisateurs pour 
générer des contenus à partir de jeux vidéo a fait d'eux le principal levier d'apprentissage de la grammaire iconique, narrative, voire cinématographique dont l'acquisition est essentielle pour faire un jeu aux normes professionnelles. De nombreux contenus vidéo-ludiques de format court circulent ainsi que le réseau, et occupent un peu la place que tiennent les courts-métrages au cinéma : ils sont une école pour jeunes créateurs.

L'objet de ce présent article est d'analyser ces dynamiques d'apprentissage et l'évolution historique de leurs limites et contraintes, en nous focalisant sur une forme où ces leviers " buissonniers " et " non professionnels » d'apprentissage des canons de la pratique atteignent leur quintessence : les films tournés et montés à partir de moteurs graphiques de jeux, appelés dans le vocabulaire indigène « machinimas ». La culture des « machinimas » est ainsi analysée comme une modalité d'entrée dans le métier du jeu vidéo. Quelles sont les modalités de conversion professionnelle des joueurs amateurs qui les fabriquent ? Quels sont les profils principaux de trajectoires ? Deux trajectoires peuvent être distinguées, celle de l'autonomisation artistique et de la consécration dans le milieu des arts plastiques contemporains et celle de la professionnalisation dans le jeu vidéo : comment cette répartition a-t-elle évolué au cours des dix dernières années?

Une définition claire des machinimas est donnée par Marino (2004) : " la création de narrations visuelles par l'enregistrement d'événements et de performances avec des personnages de synthèse créés et animés à l'aide d'un environnement virtuel ajusté issu d'un jeu vidéo ». Le mot machinima fut inventé au début de l'année 2000 par Hugh Hancock (un jeune travailleur dans les studios d'animation professionnelle à Édinbourg, qui par ailleurs était fan de Quake) pour constituer un terme générique regroupant sous un vocable unique les productions audiovisuelles faites par diverses communautés de créateurs depuis différents jeux. Cette trouvaille lexicale connut le succès car Hugh Hancock était aussi le fondateur du premier lieu de distribution fédérant sur une plate-forme convergente toutes ces occurrences hétérogènes, parmi lesquelles des enregistrements d'exploits de joueurs, betisiers, clips parodiques, formes fictionnelles, vidéos en série. En tant que technique de production, le terme se réfère au rendu d'images de synthèses au moyen de moteurs $3 D$ grand public ou ordinaires (par opposition à ceux de professionnels mais surtout par opposition à l'image pré-calculée). Le rendu peut être effectué en temps réel, en utilisant un ordinateur personnel. Mais, en tant que genre cinématographique, le terme se réfère aux films créés par les techniques 
décrites ci-dessus. En général, les machinimas sont produits en utilisant des outils (enregistrement de séquence, angles de caméra, éditeur de cartes, éditeur de scénarios, etc.) et des ressources (arrière-plans, niveaux, personnages, apparences, etc.) disponibles dans un jeu.

Notre investigation vise ainsi à comprendre le rôle joué par un lieu de partage autodidacte dans les apprentissages esthétiques de joueurs et dans leur pénétration des mondes de l'art. Il n'y a pas de convertibilité directe en «métier » de la culture des machinimas. La résistance à la conversion directe s'explique par le fait que, si ces films réalisés par certains joueurs avec leurs moteurs sont une possibilité d' « entrée dans le monde de l'art », et plus précisément dans la sphère du cinéma et dans celle des arts plastiques, cette possibilité s'appuie en même temps sur l'utilisation d'outils de basse résolution technique et qui ne sont pas au standard par rapport aux règles de l'édition professionnelle. Par exemple, alors que le cinéma d'animation utilise principalement des moteurs graphiques puissants et exigeants en temps de calcul, qui font de la 3D précalculée, les auteurs amateurs qui font des vidéos animées à partir de jeux vidéo bricolent avec des moteurs graphiques qui font de la 3D en temps réel, beaucoup moins gourmands en temps de calcul et dont le rendu de ce fait est plus pauvre plastiquement.

Pour comprendre le rôle des machinimas dans la formation artistique et professionnelle des futurs créateurs, il est nécessaire de mettre en perspective le matériau sociologique des trajectoires d'auteurs de ces créations filmiques en les replaçant dans le contexte d'une construction progressive du jeu vidéo comme un espace culturel différencié, et des relations entre cet espace et l'espace du cinéma. L'article analysera ainsi dans l'ordre les questions suivantes : tout d'abord, il s'agira d'étudier le rapport que le jeu vidéo entretient avec le cinéma. Les réalisations amateurs en effet ne peuvent être comprises si on ne situe pas leur essor dans le contexte d'une dépendance très forte du secteur du jeu vidéo, comme industrie et comme art, par rapport à l'espace du cinéma, et même d'un certain cinéma d'action très commercial et faiblement légitime. Mais à quel profil et à quelle population appartiennent ces créateurs en herbe, qu'ils se tournent vers la spécialisation professionnelle, vers l'émancipation artistique, vers la pratique d'amateurs enrichis devenus critiques, ou vers de simples expérimentations solitaires? Une seconde section nous permettra de réinscrire ces bricolages audiovisuels cumulés entre amateurs dans une histoire des «supports de patrimonialisation » permettant au jeu vidéo d'acquérir une légitimité artistique, et à leurs protagonistes d'acquérir une culture 
historique de ses productions. Nous exploiterons pour cela des données historiques et une enquête statistique commandée à l'institut Médiamétrie, et ciblée sur les utilisateurs du haut débit en France qui pratiquent le jeu vidéo. Enfin, nous chercherons à mieux comprendre l'accès différentiel, selon les catégories de population, aux principales voies de la consécration, artistique et professionnelle, dans ce secteur. Les contributeurs à l'émergence de cette nouvelle « culture numérique » peuvent-ils en vivre ? En observant l'identité des bénéficiaires de prix dans les principales instances de consécration culturelle, nous montrerons l'existence de trois trajectoires de professionnalisation des joueurs devenus créateurs. Comment se distribue l'activité entre des réalisations artistiques et des commandes alimentaires?

\section{LE RAPPORT DE SUBORDINATION ARTISTIQUE DU JEU VIDÉO PAR RAPPORT AU CINÉMA}

Le jeu vidéo subit un double processus. D'une part, un processus historique profond de dépendance du jeu vidéo au cinéma, ancré dans les origines industrielles de ce secteur. D'autre part, un processus plus récent, et lié aux créations amateur, d'affaiblissement de cette subordination au cinéma, qui se développe autour de la diffusion des « machinimas ».

\section{Le jeu vidéo dans la dépendance du cinéma}

Le jeu vidéo a toujours entretenu des relations d'inféodation par rapport à l'industrie du cinéma. Il naît en tant que réalité industrielle dans les années 1970, sous l'influence en grande partie du « Nouvel Hollywood », c'est-à-dire des gros producteurs de l'industrie cinématographique, cherchant à renouveler leurs méthodes de production parce qu'ils ont besoin de diversifier leurs revenus pour sécuriser leurs productions et leurs investissements de plus en plus lourds (Biskind, 2002). Cette stratégie du « Nouvel Hollywood», dont le jeu vidéo serait ainsi un enfant (King, 2002), répondait alors à la concurrence nouvellement introduite par l'essor de la télévision, qui depuis la fin des années 1960 conquiert une part de plus en plus importante du temps d'exposition des consommateurs devant les écrans. Elle visait aussi à prendre en compte une transformation importante du public jeune et adolescent, devenu plus éduqué du fait de la massification de l'enseignement sur cette période, et s'affirmant depuis lors comme quémandeur de divertissements plus évolués, plus à même de capter une attention devenue plus fluctuante, et de plus en 
plus focalisée sur les sortilèges de l'innovation technologique (Bruno, 1993). Un exemple emblématique de cette "naissance » industrielle du jeu vidéo est l'achat, à l'automne 1976, de la petite entreprise pionnière du milieu du jeu vidéo, Atari, par le conglomérat des médias et des industries du divertissement Warner Communications Inc, où elle va rejoindre, dans la branche « entertainment» de ce géant du film, le département de comics et la filiale discographique. En 1980, Atari représente déjà $30 \%$ du chiffre d'affaires global du conglomérat Warner.

À partir de cette naissance industrielle datant des années 1970, le jeu vidéo suit ensuite trois phases successives de soumission à de fortes contraintes industrielles. C'est précisément cette forte industrialisation qui va fixer la dépendance de ses productions par rapport à l'espace cinématographique. L'étape initiale de cette industrialisation des processus de fabrication " logicielle » fut la réponse apportée par le secteur, sous la houlette de la firme Nintendo, à la très grave crise de 1983, qui était l'exemple même d'une crise de surproduction (Genvo, 2009) due à l'avalanche sur le marché d'œuvres autoproduites, de mauvaise qualité, fabriquées par des amateurs et qui ont détourné le public. Nintendo, qui sera suivi par Sega, l'autre tenant de ce marché industriel naissant, prend la décision de contrôler sévèrement la création logicielle en prenant le contrôle de la plate-forme éditoriale, purgeant le marché d'un grand nombre de productions indépendantes et éliminant progressivement la production amateur de l'écoulement sur le marché. La logique d'industrialisation reposait ainsi sur un malthusianisme éditorial, sur une sélection drastique des éditeurs de jeux qui est directement opérée et contrôlée par le constructeur de console. De plus, au-delà du fait qu'il reposait sur le monopole de la fabrication des cartouches ${ }^{2}$, en amont, et sur le monopole de la distribution, en aval, l'émergence du modèle industriel était caractérisée par une inversion des rapports de force entre éditeur et distributeurs, jusque-là très favorables aux distributeurs, dans la filiation de l'histoire du jouet : le " consignement », qui était jusqu'en 1983 pratiqué, reposait sur des accords très avantageux pour le distributeur, faisant que celui-ci ne prenait pas à sa charge les invendus ; cela avait abouti à la multiplication de produits devant être vendus très vite, avec un écoulement de stocks en cassant les prix qui ne permettait pas à des productions de qualité d'émerger.

2. Le constructeur Nintendo calqua ses normes de qualité, comme le taux de rejet de ses matériels, sur celles du secteur des magnétoscopes (Blanchet, 2010, p. 191). 
L'industrialisation s'intensifie dans les années 1990 avec comme caractéristique une hausse continuelle des coûts fixes, due à la complexification que la non-standardisation des équipements généra en alourdissant le développement informatique. À partir de 1987, les éditeurs doivent ainsi s'adapter à une prolifération de plates-formes de jeu et de standards ; ils sont obligés d'organiser leurs équipes de manière à pouvoir éditer un même titre sur différentes machines. La complexification des plates-formes a aussi entraîné l'augmentation des salaires pour rémunérer des employés toujours mieux formés.

Enfin, l'industrialisation est aussi liée à la hausse des frais de distribution qui est liée à la diffusion d'ampleur continentale des équipements et des produits. La distribution au départ n'était pas directement mondiale, la politique choisie par les Japonais leaders du marché en 1983-2006 ayant durablement structuré une partition du marché en trois zones : Japon, Amérique du Nord, Europe. Cependant, à partir de 2006, la commercialisation simultanée des produits (jeux et consoles) sur l'ensemble du globe devient petit à petit la norme de distribution. Apparaît en effet alors dans le jeu vidéo la stratégie du « widespread opening » initiée dans les marchés du cinéma dans les années 1990 (notamment pour éviter le piratage) : la Wii, par exemple, est commercialisée simultanément en 2006 en l'espace de quatre semaines seulement (Blanchet, 2010 , p. 285). Les jeux, depuis 2005, sont systématiquement sortis mondialement : une tradition s'est imposée qui fait que l'industrie a institué un jour de sortie des nouveautés sur les trois grands marchés : le lundi aux États-Unis, le jeudi au Japon, le vendredi en Europe.

La conséquence de la forte industrialisation et du poids important des coûts fixes est la prédilection des producteurs de jeux vidéo pour les stratégies de « prévente » (presold strategies), qui consistent à proposer des jeux dont les contenus ont déjà connu précédemment une popularité au cinéma, afin de rendre plus probable le succès de l'adaptation. Ainsi, les jeux cherchent à acheter de très nombreuses licences d'exploitation cinématographiques ou à s'inspirer librement d'autres films.

Certes, de tout temps, le jeu vidéo a cherché son inspiration dans le cinéma, notamment par une stratégie d'emprunt subtil. Par exemple, le premier succès de l'ère industrielle du jeu vidéo, Mario, qui est né dans Donkey Kong, puise ses références dans la tradition du film burlesque américain, dans celle du cartoon, et fait directement référence, à travers la figure du singe géant posé au sommet d'un bâtiment, à des scènes du film King Kong de 1933. 
De même, la célèbre série Grand Theft Auto de Rockstar (dont le premier opus sort en 1997) s'inspire d'une tradition cinématographique, effectuant des emprunts à L'Enfer est à lui de Raoul Walsh (1949) aussi bien qu'à Scarface de Brian de Palma (le héros de l'épisode de 2002 «Vice City » de cette série de jeux vidéo, Tommy Vercetti, est une référence directe au tueur à gage de ce dernier film, Tonny Montana). À ces références culturelles s'ajoute, initiée par des éditeurs pionniers depuis 1983, une logique d'achat systématique de licences cinématographiques. Alors que, sur les 15000 titres de jeux vidéo sortis depuis 1975 il n'y en a que $15 \%$ qui sont issus d'un film commercial, ce pourcentage s'élève à plus de $30 \%$ après 1995 (Blanchet, 2011). Par exemple, en 1998, Electronic Arts, qui misait précédemment surtout sur des franchises propres (comme « Les Sims ») et sportives (grâce à des accords avec les ligues professionnelles du football et du basket, par exemple), achète la licence Harry Potter (et sort tous les jeux simultanément à leur sortie sur écran dans les années 2000), celle sur le film Le Seigneur des Anneaux en 2001, en complétant ces achats par l'acquisition des droits sur l'œuvre romanesque. Activision, troisième éditeur sur le marché mondial, rachète en 2000 de nombreuses licences à Disney, signant notamment un accord d'exclusivité sur les films d'animations en images de synthèse produits par DreamWorks, et à Marvel, ce qui lui permet de se focaliser sur les jeux adaptés de super-héros. Dans ce nouveau paysage de dépendance du jeu vidéo par rapport aux contenus cinématographiques, les jeux vidéo sortent simultanément aux films ${ }^{3}$ : le jeu Harry Potter est disponible le 15 novembre 2001 dans les rayons des revendeurs alors que la sortie du film aux États-Unis est fixée au 16 novembre (le 5 décembre 2001 pour la France). Electronic Arts propose même les versions vidéoludiques toujours légèrement en amont à leur sortie en salle (Blanchet, 2010, p. 323). Même les firmes qui ne sont pas leaders sur leur marché concentrent leurs investissements sur l'achat de licences cinématographiques. Ubisoft, qui naît en 1986, et qui est jusqu'en 2008 un « outsider » sur le marché, acquiert dans les années 1990 la franchise pour la distribution en Europe sur Star Wars et Indiana Jones, et, en 2000, sur des séries télévisées (Les Experts, Lost) voire sur certains blockbusters comme King Kong en 2005 et surtout Avatar en $2009^{4}$. Selon la société d'étude de marché NPD Group,

3. C'est une tendance qui a démarré avec Batman en 1989, premier exemple de simultanéité du processus d'édition du jeu avec la première exploitation en salle du film adapté. Cela introduit une nouvelle période, où il n'est plus rare que les joueurs voient s'afficher pendant qu'ils chargent le jeu des écrans affichant la sortie ou la disponibilité en vidéocassette du film adapté.

4. Il semble que, dans le cas d'Ubisoft, c'est non pas des considérations financières (elle ne pouvait pas rivaliser) mais la réputation de son équipe de développeurs, autour de Michel Ancel, qui a fait qu'elle reçut quelques licences pour exploitation de certains blockbusters 
plus de la moitié des vingt jeux les plus vendus aux États-Unis pour les fêtes de fin d'année de 2003 étaient, tous supports confondus, des suites ou dérivés de titres existants. Le nombre des créations originales parmi les jeux les plus vendus n'a cessé de décliner continuellement à partir des années 1990.

Les synergies entre jeu vidéo et cinéma ont ainsi été amorcées, dans un contexte malthusien en réaction à la crise du modèle de prolifération de contenus amateurs de 1983, par des stratégies de prévente, misant sur la popularité d'un contenu déjà existant qui assure qu'il soit vendu facilement. Cela a entraîné l'intériorisation massive par les jeux vidéo de contenus cinématographiques surtout venus du cinéma d'action et d'animation. Une radicalisation de cette dépendance a marginalement lieu depuis la fin des années 1990, avec l'internalisation dans les studios de développement des éditeurs de jeu vidéo d'équipes cinématographiques qui font des séquences en prise de vue réelle, qui seront intégrées dans les cinématiques du jeu vidéo, et parfois aussi servent à la livraison des films ${ }^{5}$. Cependant, les séquences en prise de vue réelle sont assez rares dans les cinématiques de jeux vidéo car la tendance est à privilégier les images de synthèse pré-calculées.

\section{Une assimilation du jeu vidéo au cinéma le moins légitime}

La synergie entre jeu vidéo et cinéma touche un segment tout à fait particulier de l'espace du cinéma : les films les moins légitimes et les plus commerciaux. Si l'on prend comme moyen de notre démonstration l'identification du statut, auprès de la critique, des films qui ont été tirés de jeux vidéo, et du genre auquel ils appartiennent, on observe une propriété très remarquable.

Si l'on se focalise ainsi sur l'exemple des jeux vidéo adaptés ensuite en film, une vingtaine de jeux vidéo sont concernés par le fait qu'ils ont été l'objet d'une adaptation tournée en « prise de vue réelle » en collaboration avec Hollywood

\footnotetext{
5. Ainsi, le studio Ubisoft de Montréal a présenté à Cameron des modélisations d'éléments de jeu qui ont été intégrés dans le film (Blanchet, 2010, p. 346-347); de même, Ubisoft s'oriente vers la production d'images de synthèse pour le cinéma et toute forme de programme individuelle en faisant là de lourds investissements, et c'est ainsi qu'Ubisoft sort en 2009 Assassin's Creed : trois courts-métrages diffusés sur Internet puis en deuxième partie de soirée sur la chaîne NRJ12 qui visent à développer l'univers du jeu qui se déroule dans l'Italie de la Renaissance.
} 
entre 1993 et 2000 (Augros, 1996) ${ }^{6}$. Les films en question ont eu des postérités commerciales très contrastées, certains, rares, ayant été des succès, la plupart ayant été des échecs. Ils ont néanmoins trois caractéristiques communes. D'une part, ils sont l'œuvre de réalisateurs plutôt débutants. On ne trouve parmi eux aucun réalisateur ayant déjà dirigé un «blockbuster » : les adaptations de jeux vidéo sont notamment grandes consommatrices de cinéastes étrangers débutant dans le système de production hollywoodien, tel Christophe Gans qui réalise son premier film avec Silent Hill (2006) ; ou l'Anglais Simon West qui n'avait précédemment à son actif qu'un blockbuster estival produit par Jerry Bruckheimer, Con Air (1997). D'autre part, on trouve parmi leurs acteurs peu de stars de cinéma et dans le meilleur des cas des «stars en devenir », comme c'est le cas pour le film Lara Croft qui fut rendu célèbre par la présence à l'écran d'Angelina Jolie. Mais cette dernière accéda au statut de star hollywoodienne grâce à la visibilité du film. Enfin, les productions sont souvent des runaway productions, autrement dit des productions cofinancées par Hollywood et des investisseurs étrangers, et elles sont d'envergure moyenne, les tournages ayant lieu au Canada ou en Europe, afin d'en réduire les coûts et d'étaler sur des chaînes câblées ou des vidéoclubs le film. Cette synergie entre jeu vidéo et ce segment très particulier de l'espace du cinéma : les films les moins légitimes et les plus commerciaux, indique le relatif manque de légitimité du jeu vidéo et de tout ce qui touche au jeu vidéo dans l'espace cinématographique.

\section{L'essor des machinimas à partir de 2008 et l'autonomisation cinématographique du jeu vidéo}

Cependant, à partir de 2008, et du fait de l'apparition des créations amateurs appelées " machinimas », un certain brouillage de ce lien de subordination artistique apparaît. Trois principaux jalons scandent le processus de progression de la diffusion auprès d'un plus large public des machinimas. En 2000, grâce à une invention des éditeurs de Quake, il commence à être possible de livrer les machinimas dans un format de fichier vidéo conventionnel, alors que les précédents étaient des fichiers « algorithmiques », qui requéraient pour être visionnés d'être lancés à l'intérieur du jeu original ${ }^{7}$. Puis, en 2003,

6. Ces films sont les suivants : Wing Commander 1999, Lara Croft : Tomb Raider 2000, Final Fantasy 2001, Super Mario Bros 2003, Resident Evil 2004, Mortal Kombat 2005, Silent Hill 2006, Street Fighter 2008, Prince of Persia 2009, Les Sables oubliés 2010.

7. Quad God, tournée avec Quake III Arena fut le premier machinima à avoir été diffusé dans un format vidéo conventionnel, au lieu d'être un fichier dit « démo » nécessitant que, pour 
tirant parti de ces innovations imprévues venues des utilisateurs, les éditeurs de jeu intègrent par défaut des options éditoriales (mouvements de caméra) et des options de montage dans leurs jeux vidéo, afin de faciliter une « jouabilité émergente » et de capturer un ressort supplémentaire d'engagement ${ }^{8}$. Ces éditeurs firent ainsi des machinimas un exemple remarquable, dans le secteur artistique, d'innovations horizontales (von Hippel, 2005) voire, plus précisément dans le cas présent, ascendantes (Cardon, 2005). Enfin, en 2008, la commodité d'accès à ces moteurs de jeux se renforce encore avec leur autonomisation et leur extériorisation hors des environnements ludiques dans lesquels ils avaient été conçus. À partir de cette date est ainsi forgé le modèle de la « DIY (Do It Yourself) animation ». Des logiciels comme Machinimation, Moviestorm, Muvizu sont ainsi spécifiquement conçus pour réaliser des machinimas : ils intègrent un moteur de jeux et un logiciel de montage. Ces logiciels exploitent des licences de moteurs de jeux vidéo (ainsi, Muvizu est l'extériorisation du moteur du jeu Unreal Engine, et Movistorm celle du jeu de simulation The Movies).

À partir de cette date, les usages se démultiplient principalement dans deux secteurs. Tout d'abord, certains groupes musicaux font appel aux machinimas pour baisser les coûts de création d'animations vidéo. Ils sont utilisés comme des alternatives à bas coût, notamment par certains groupes en début de carrière ${ }^{9}$. Une émission de télévision sur MTV, entre 2003 et 2005, uniquement consacrée à la diffusion de clips vidéo en machinimas, popularisera

l'afficher, l'ordinateur le recompile avec le code du jeu originel. Un tel basculement vers les médias conventionnels a offert aux producteurs de ces films une audience plus large et a ainsi lancé la reconnaissance pour cette forme de création. Matt Kelland, Dave Morris et Dave Lloyd ont ainsi considéré la diffusion de Quad God comme « a key moment in the development of machinima » (cité in Nitsche, 2005).

8. Ayant ainsi pris conscience de tout le potentiel du machinima, Epic Games, l'éditeur de Unreal Tournament 2003, inclut, pionnier en la matière, un outil appelé Matinee avec le jeu, et sponsorisa un concours (offrant un prix de 50000 dollars) de création de film machinima avec le jeu. Auparavant, il y avait certes des paramètres dans les jeux pour « enregistrer des séances de jeu du point de vue subjectif du gamer ", essentiellement dans une perspective d'entraînement sportif, pour aider les joueurs à visionner leurs performances et leur apprendre à progresser. Mais elles étaient limitées, et IdSoftware leur avait même donné un coup d'arrêt en décembre 1999, lors de la sortie de la version 3 de Quake, la firme ayant durci ses conditions d'utilisation du code réseau.

9. Le premier groupe de musique actuelle ayant composé son clip en machinima est probablement Vice Romania, qui le fit en novembre 2008. Leur vidéo, This Is It fut créée par Lucinda McNary du groupe amateur Two Moon Graphics, du Kansas. La création additionnait l'usage du moteur Moviestorm et du logiciel de montage 3D DAZ3D. 
cette tendance sur deux saisons. D'autre part, des usages importants se développent dans la communication d'entreprise et institutionnelle : certaines agences de marketing et de communication utilisent des machinimas pour le pitch de leurs idées à des clients prospectés, d'autres directement composent leurs créations en utilisant cette méthode d'animation, ce qui est une solution pertinente lorsque le public visé appartient aux nouvelles générations et est susceptible d'être fréquemment exposé à l'usage de jeux vidéo.

En revanche, l'utilisation des machinimas reste encore très rare, bien qu'elle commence à apparaître, dans deux autres secteurs. Elle commence à émerger dans les sphères de production professionnelles cinématographiques et télévisuelles ; le principal exemple à ce sujet est la série télévisée d'animation pour enfants Jacks and Holly, à la télévision britannique. Enfin, une finalité de prévisualisation se constitue, qui est utilisée dans l'univers professionnel du cinéma et de la télévision. Par exemple, Moviestorm est de plus en plus utilisé, depuis 2009, comme outil de préproduction, pour visualiser le storyboarding dans des productions en tournage et en prise de vue réelles. La facilité de réalisation de storyboards avec Moviestorm a ainsi amené $\mathrm{L}$. Watson, le réalisateur de The Letter, à l'utiliser pour son storyboard. De même, le scénariste londonien Dean Wells mentionne l'utiliser régulièrement pour tester ses idées de films et publier des trailers basés sur ses scripts, destinés à convaincre les producteurs et d'autres financeurs de la profession.

\section{LE JEU VIDÉO COMME ESPACE CULTUREL DIFFÉRENCIÉ}

Si le jeu vidéo dans son ensemble peut être décrit comme fortement articulé au cinéma d'action, comme on vient de le voir, la modalité de cet ancrage dépend d'une polarité interne à son espace culturel (2.1). D'autre part, cette articulation a évolué depuis 2000 vers une différenciation et une séparation des circuits de formation professionnelle des deux filières. Dans un tel contexte, la pratique et la lecture des machinimas constituent une des modalités centrales de l'apprentissage, pour les futurs professionnels du secteur du jeu vidéo, des grammaires du cinéma (2.2).

Une opposition structurante apparaît, dès les années pionnières du jeu vidéo (1983-1993), entre un pôle marqué par des usages ouverts et créatifs, et caractérisé par des utilisateurs plus âgés, ayant plus de degrés de liberté dans l'usage, et un pôle " de produits à consommation plus passive », caractéri- 
sée par des utilisateurs très jeunes, dont les parcours d'usage sont bridés et canalisés par des produits se prêtant peu au détournement et à la créativité. Entre 1983 et 1993, dates des deux crises qu'a connues le secteur (Genvo, 2008), une opposition symbolique forte passe alors entre Nintendo d'une part, qui a fait délibérément le choix d'une orientation éditoriale tournée vers les enfants et dont le cœur de cible est entre 6 et 8 ans, et Sega, d'autre part, qui visait plutôt un public d'adolescents, dont le cœur de cible était entre 10 et 16 ans, mais qui « ne cherchaient pas des produits qui leur rappellent l'époque où ils avaient 6/8 ans. Au contraire, ils souhaitaient plutôt ressembler aux 18/25 ans » (Blanchet, 2010). Sur le pôle " enfantin », les firmes et les éditeurs se trouvent obligés, du fait de s'adresser à des enfants, à construire des formes de divertissement très contrôlées, initiées aux États-Unis par la Walt Disney Company. De l'autre, autour de la coque noir jais de la console MegaDrive, commercialisée sous le nom programmatique de Genesis aux États-Unis à partir de 1988, le jeu vidéo se trouve assimilé à toute une série d'identifiants high-tech : le motif en forme de cercle rappelle le compact-disc au-dessus du châssis, il y a une prise jack pour un casque audio, de nombreux partenariats d'exclusivité sont signés avec des stars musicales (comme Michael Jackson, dont le film Moonwalker est adapté sur Sega) et des studios de cinéma.

Mais c'est surtout dans la période après 1993, avec l'apparition de Microsoft et de Sony dans cet espace, que s'est creusée cette polarité. Un pôle de la pratique - autour de Sony, de Microsoft, d'éditeurs typiques comme Blizzard ou Rockstar Games - est orienté vers des usages plus créatifs et promeut un type d'usage conçu comme « jeu ouvert et sans fin », qui a été rendu possible par le développement massif de la connexion à l'Internet haut débit des jeux vidéo et des consoles à partir de 2004. Les analystes de l'espace du jeu vidéo en sciences de l'information et de la communication multiplient d'ailleurs un biais de focalisation sur ces pratiques fortement légitimées par le milieu éducatif et valorisantes : par exemple, Celia Pearce (2003) parle de « co-éditique », Franck Beau (2006) souligne l'essor de ce qu'il appelle, faute de mieux, « craftware », dans le monde du jeu vidéo.

Sony arrive sur le marché en 1994, et Microsoft en 1995. Leur arrivée déclenche des orientations techniques qui seront marquantes pour ce nouveau pôle et constitueront un clivage. D'une part, ils prolongent le mouvement de " convergence » initié par Sega dans la décennie précédente, en forçant à l'adoption, par le jeu vidéo, du support optique (le CD-ROM et le Blue-Ray), et en rendant incontournable l'usage par les jeux vidéo de moteurs graphiques 
permettant de générer en temps réel des représentations en 3D. Mais, surtout, ils introduisent dans l'expérience du jeu vidéo la connectivité haut débit, assurée par ces nouvelles consoles et notamment par l'expérience acquise par Microsoft dans les cartes réseau et la maîtrise des standards techniques de communication (comme Ethernet). Les consoles de ces deux constructeurs permettent non seulement de jouer à des jeux mais de télécharger ou de visionner et partager des films et des musiques; elles sont d'ailleurs vendues, selon un geste de marketing commun et spectaculaire, en bundle avec des DVD de films à succès récents (Augros, 1996). C'est ainsi autour d'un titre développé en lien avec Microsoft dès 1993, Doom, que le PC est consacré par les médias et les analystes comme machine ouverte à des usages créatifs et suscitant des créations collectives. Ce titre a établi les fondements d'une nouvelle façon de faire du jeu, à la première personne mais en réseau, et autour de cette connectivité il a initié une révolution interne. Parce que le jeu se jouait en réseau, sa circulation et la rémunération de ses concepteurs étaient organisées autour d'un nouveau mode de distribution : alors que précédemment les jeux connaissant une large diffusion étaient acheminés chez les revendeurs par des distributeurs qui traitaient avec les maisons d'édition, Doom, conçu par une petite équipe de développement, était à l'inverse au départ gratuit et téléchargeable directement sur Internet. Seuls les premiers labyrinthes étaient fournis : si le joueur voulait en voir plus, il devait passer commande directement chez l'éditeur pour pouvoir télécharger les autres épisodes (Genvo, 2009, p. 47). Microsoft proposa même de tirer du réseau des revenus supplémentaires pour le constructeur en proposant aux joueurs la possibilité d'un abonnement mensuel à sa console, le Xbox Live (permettant d'affronter à distance d'autres joueurs par le monde). En 2001, un autre jeu emblématique est conçu, sous le contrôle éditorial de Microsoft, d'abord sur console alors qu'il provient d'un genre développé sur et pour les micro-ordinateurs personnels : il s'agit de Halo.

\section{La place minoritaire du pôle « ouvert »}

Sony et Microsoft sont donc, à partir de 2005, les pôles ouverts de l'espace industriel et culturel du jeu vidéo, attirant un nouveau public plus adulte, plus connecté, plus cultivé, plus diplômé et plus « partageur ». Ils proposent des jeux permettant de s'associer avec d'autres joueurs pour parcourir un monde fictionnel persistant et remplir des missions diverses et variées afin d'obtenir des points d'expérience, objets et nouvelles capacités. Cette position, fortement située et distinctive à l'intérieur de l'espace culturel du jeu vidéo, 
exploite la valeur symbolique de produits qui sont identifiés, du fait de leur plasticité et de leur ouverture, à une « sand box » (bac à sable). À partir de 2005 avec l'essor du Web dit 2.0 et de l'User Generated Content, qui a renforcé la base d'utilisateurs possible pour le succès de tels produits ouverts, s'est radicalisée la diffusion de jeux vidéo cassant la linéarité et le déterminisme du récit, comme le jeu en ligne à succès World of Warcraft (Blizzard, 2005). Les joueurs de ce pôle d'usages pouvaient se voir offrir des modes d'exploration encore plus ouverts, et on leur proposait une déambulation sans but précis. Il se construisit alors une figure, hautement valorisée, de l'usager coproducteur : usager invité à des fan faires, et duquel on utilisait les retours d'expérience. Des éditeurs de certains de ces jeux ont même développé des concours sponsorisés par des partenaires industriels (dans la mode pour le jeu Les Sims ou dans l'automobile pour The Movies) : ceux-ci peuvent sponsoriser des contenus faits par des joueurs avec les possibilités de ces jeux; ils peuvent les commercialiser ensuite ou les utiliser gratuitement; en échange, ils doivent payer une somme forfaitaire pour ce geste de partenariat ${ }^{10}$.

Alors qu'il est fortement valorisé par les médias et surexposé aux enquêtes en marketing et en sciences humaines, le pôle du jeu vidéo connecté où le joueur, selon la formule du concepteur des Sims Will Smith, "produit son propre divertissement », ne représente que $40 \%$ du chiffre d'affaires du jeu vidéo. À l'autre pôle, particulièrement présent dans les jeux sur consoles portables, mais aussi dans de nombreux jeux sur console, les produits proposés sont fermés. Ils offrent certes à l'usager le contrôle sur le choix des « branches » de l'intrigue (Murray 1997) mais ils structurent l'attention du consommateur sur un fil narratif qui reste prégnant. La captation de l'attention du joueur fait d'ailleurs l'objet des principales ruses des traités de « game design » (Guardiola, 2005) ${ }^{11}$. Les jeux sont difficilement transformables ou personnalisables. Du fait de la volonté de

10. Certains jeux, à l'extrémité du pôle, permettent à l'utilisateur de générer non pas des objets et des contenus mais littéralement des « niveaux de jeux » supplémentaires. Cette production peut ensuite être partagée avec d'autres joueurs en utilisation locale ou au moyen d'une mise à disposition par le réseau Internet.

11. Un exemple emblématique de ce pôle plus fermé aux détournements d'usage est la célèbre série des Supermario sur Nintendo. Les jeux Mario en deux dimensions n'apportent que très peu de possibilités de détournement (le personnage ne peut qu'avancer à gauche ou à droite, et sauter), et même les jeux en trois dimensions offrent très peu d'interactions avec d'autres personnages. Ils sont si peu ouverts aux braconnages créatifs (de Certeau, 1986) que les utilisateurs qui souhaitent les modifier sont obligés de recréer des graphismes à partir de zéro pour ensuite simuler l'environnement du jeu, voire de transposer l'univers de Mario dans la réalité (comme par exemple en customisant des karts, en se déguisant ou en se filmant en train de faire la course dans la rue). 
contrôler la publication, des informations relatives au copyright s'activent sur les supports au lancement de chaque session, et sont indispensables pour les faire fonctionner. Les possibilités de création sont réduites. À cette opposition en termes de pratiques culturelles, entre ces deux mondes, s'ajoute un effet de ségrégation sociale qui pèse sur la plupart des usages connectés, dans la mesure où leur réalisation repose sur une contrainte d'abonnement Internet haut débit et le plus souvent d'équipement en micro-ordinateurs assez puissants. Les « machinimas » se réalisent en effet essentiellement dans un segment de marché très particulier de l'industrie du jeu vidéo : celui lié au micro-ordinateur (elles sont ainsi très fréquentes, à leur démarrage, sur les grands succès des jeux sur micro-ordinateurs : Doom, le jeu vidéo sur PC de 1993 qui a fait naître ce segment de marché en 1993, Quake paru lui en 1996, Half-Life créé en 1998 et Halo en 2001).

\section{Encadré : une enquête statistique sur la place des pratiques créatives auprès des consommateurs de jeu vidéo}

Nous avons voulu vérifier l'origine culturelle et sociale de ces pratiques de détournement et tester si elles étaient minoritaires ou pas, en nous focalisant sur la population des consommateurs des offres de haut débit. Une enquête, administrée par Mediamétrie, a ainsi été menée sur 1000 abonnés au très haut débit (abonnés Très Haut Débit Fiber To The Home en Îlede-France), parmi lesquels 615 ont affirmé « jouer au jeu vidéo » et 310 ont répondu au questionnaire. Il en ressort le constat suivant : alors que, parmi les joueurs de jeux vidéo abonnés au très haut débit, les pratiques de personnalisation sont légèrement majoritaires $(59,7 \%$ ont modifié le paramétrage par défaut de leur jeu, 52,3\% déclarent s'être procurés une extension, soit en l'achetant auprès du constructeur, soit en la téléchargeant sur des sites de joueurs), la réalisation de machinimas et de performances artistiques concerne une frange très marginale de la population. $3,1 \%$ des joueurs qui reconfigurent leur jeu vidéo seulement font des vidéos à partir des jeux (soit environ 2,0 \% de l'ensemble de des joueurs). La réalisation de machinimas est une pratique d'ailleurs encore plus rare que la réalisation de détournements informatiques comme les « mods », qui concernent eux 19,8\% du total des joueurs, les musiques « cheaptunes » ( 8 bits), qui concernent $14,3 \%$ des joueurs, ou encore l'écriture de textes sur les jeux vidéo $(3,9 \%)$, et la fabrication de romans-photos et de bandes dessinées $(2,8 \%)$. La composition de films et clips d'animation graphique à partir des jeux reste une pratique ciblant un public extrêmement marginal. 


\section{L'absence de passerelle professionnelle entre jeu vidéo et cinéma d'animation}

Faire un jeu vidéo suppose de croiser des compétences en animation, en cinéma et en gameplay. Pendant longtemps, ces compétences diverses étaient acquises grâce au fait que les principaux créateurs avaient des parcours transverses. Ainsi en France, jusqu'à vers 2005, date à laquelle se sont multipliées les offres professionnelles de formation aux métiers du jeu vidéo, faisant que la majorité des chefs de projet recrutés depuis cette date sont issus d'écoles spécialisées, les créateurs étaient essentiellement autodidactes, et surtout venaient d'autres secteurs. Ils venaient de la musique ( $\mathrm{Ph}$. Ulrich, D. Cage), du graphisme (Éric Chahi, auteur d'Another World et Heart of Darkness) ; du dessin animé (Éric Viennot, auteur de l'Album secret de l'oncle Ernest, et devenu ensuite directeur de création chez Lexis Numérique), de la littérature... Ils ont alors, dans le jeu vidéo, inventé les canons esthétiques du secteur au fil de deux décennies de création. Depuis 2005, la multiplication des écoles spécialisées et l'industrialisation du secteur a entraîné une sévère division du travail et la fin du profil de «super-développeur » ayant touché à plusieurs parcours de création. Il s'ensuit une certaine crise de créativité, reconnue par les acteurs, et qui va de pair avec la crise économique que subissent particulièrement les studios indépendants, par opposition à la relative bonne santé des gros éditeurs et des jeunes pousses à petits budgets faisant de petits jeux gratuits ou sur les réseaux sociaux.

La réalisation de machinimas, dans ce contexte, a vu sa signification s'inverser. Elle n'a jamais été utilisée pour devenir cinéaste d'animation. Les auteurs de machinimas n'ont aucune compétence propre aux métiers de l'animation professionnelle et n'utilisent pas les mêmes outils ${ }^{12}$. Les rares studios qui développent des productions à base de machinimas le font dans les créneaux très commerciaux de la communication institutionnelle ou de la publicité pour des produits de haute technologie (les machinimas étant reconnus par les publics de consommateurs de ces produits). Ces studios, rares, font le choix de l'indépendance. La prédilection pour fonder des studios indépendants (plutôt que de s'insérer dans des équipes déjà constituées d'animation) est une conséquence de la rareté des passerelles professionnelles. La modalité commerciale majoritairement choisie est l'abonnement de faible valeur pour avoir accès " en primeur » aux vidéos. Par exemple, Rooster Teeth Productions

12. Krögh et al. (2010) signalent en 2006 deux exceptions : Spike TV et MTV. 
réservait le vendredi la primauté de ses épisodes à ses abonnés, et, le lundi, il diffusait les contenus gratuitement auprès de tous. Mais, à la saison 5 de leur série principale (Red vs. Blue), au bout de 30 minutes de diffusion auprès des abonnés, il y avait déjà plus de 50 commentaires moyens par des utilisateurs uniques, ce qui témoigne de courts cycles de consommation, synchronisés avec le moment de la production (Haefliger et al., 2009).

Il y avait, en 2006, sept principales compagnies qui développaient une activité commerciale autour de la vente de films d'animation faits avec des moteurs 3D de jeux. Ces sept compagnies étaient toutes passées, dans une phase précédente, par la production amateur de films gratuits et avaient toutes eu des films primés dans des festivals comme le festival « académique » (Krögh et al., 2010). Elles diffusaient leurs œuvres en déposant les contenus sur des sites dont elles louaient l'espace auprès d'hébergeurs. Un mouvement déclencheur de l'entrée vers la commercialisation de ces compagnies était lié au fait qu'elles ont été confrontées, au fil de la popularité acquise par leurs œuvres, à l'élévation exponentielle de la bande passante, et à des coûts subitement plus élevés d'hébergement ${ }^{13}$. La popularité pouvait être très coûteuse, en 2006, et les amateurs déployaient des combines pour fabriquer des produits dérivés. Les plates-formes vidéo (comme YouTube ou Rever) en émergent vers 2005, changent la donne en abaissant les coûts et en permettant de rester plus longtemps dans la circulation non commerciale.

Dans un tel contexte de rareté des passerelles professionnelles, les machinimas ont plutôt un rôle, dans les premières années de formation à la pratique professionnelle, de sensibilisation à la grammaire du cinéma - du cadrage, du montage. Tel machinima pourra être un moyen de s'entraîner à réaliser un bon « raccord de regard » (dont on sait l'importance pour la qualité de l'expérience d'un jeu vidéo), tel autre pourra permettre à son auteur, et à ses commentateurs attentifs, de maîtriser les diverses techniques d' " ocularisation », tel autre encore de s'entraîner à maîtriser la façon dont le fait de faire varier les " points d'écoute », les lieux où sont captés les sons, modifie l'expérience du joueur. La plupart des jeux empruntent au savoir-faire cinématographique

13. «Malheureusement, plus vous êtes populaires plus vous devez payer de la bande passante pour que vos spectateurs soient en mesure de regarder votre série. (...) Quand nous avons commencé, nous n'avions pas assez de place pour nos vidéos. (...) Par conséquent, vers juin 2003, je me souviens que c'était à l'épisode 13 de notre série Red vs. Blue, j'ai lancé un fil de discussion dans le forum : "Si je fais un T-shirt avec le logo Red. Vs Blue, est-ce que vous l'achèteriez, pour nous aider ?". Et la réponse a été un plébiscite. J'ai donc lancé la fabrication des T-shirt, et les gens les ont achetés en grand nombre » (Krögh et al., 2010, p. 1204). 
de manière subtile pour hiérarchiser leurs éléments ou structurer le parcours $\mathrm{du}$ « spectateur », et les petites animations faciles à réaliser sont un moyen de plus en plus utilisé pour réaliser, de manière souple et peu coûteuse, les entraînements que nécessitent ces apprentissages.

\section{DEUX TRAJECTOIRES DE CONSÉCRATION SOUMISES À DES CONTRAINTES}

Dans cette section, nous donnons quelques éléments, parmi ces auteurs, sur les principales voies de la consécration, artistique et professionnelle. Nous partons pour cela d'un corpus particulier, que nous nommons " académique ", et qui est constitué par la collection de 572 machinimas destinée à la patrimonialisation du secteur, initialement collectée par H. Lowood, directeur de la Stanford Humanities Laboratory, pour la " préservation des mondes virtuels » (Lowood 2004). Une trentaine de ces œuvres sont francophones. Il nous permet de dégager une liste de 520 auteurs dont nous essayons d'observer s'ils s'inscrivent dans une trajectoire de professionnalisation. Nous avons complété par ailleurs cette analyse par une vingtaine d'entretiens biographiques avec les auteurs présents dans ce corpus, permettant de retracer les formes de consécration dont ils ont bénéficié et de dégager ainsi trois voies majeures de professionnalisation.

\section{Des formes de consécration artistique valorisant fortement les artistes diplômés}

Depuis 2010, à cheval entre l'univers du cinéma et celui du jeu vidéo, se sont multipliées les occasions de consécration pour les auteurs de machinimas. Ces instances de consécration sont « mixtes » et diverses.

Historiquement, c'est en 2002 que se crée le festival de référence, central et commun à plusieurs jeux vidéo, dans le cadre de l'Academy of Machinima Arts and Science. Il est fondé collégialement par les principaux auteurs de l'époque et sponsorisé par des constructeurs d'équipements ; il reçoit depuis, chaque année, plusieurs milliers de productions, dont le site Web correspondant à ce festival hiérarchise la visibilité selon les différentes catégories de prix attribuées. Conçu sur le territoire français, l'autre grand festival entièrement dédié aux machinimas est le «Atopic Film Festival », créé par M. Balzerani en 2009 et exclusivement consacré à eux depuis 2010. Le jury de ce 
festival se compose, en proportions similaires, de créateurs reconnus de jeu vidéo, de professionnels des nouvelles technologies et de critiques et organisateurs de festivals de cinéma.

Les machinimas apparaissent ensuite progressivement dans des festivals organisés autour par des écoles d'art et des institutions muséales. En France, comme dans la plupart des autres pays, c'est en 2005 qu'une première institution muséale reconnaît les machinimas : le Centre Beaubourg Pompidou, à l'intérieur d'un cycle de programmation qui avait déjà deux ans, "Plasticiens du Web ». Parmi les institutions muséales liées à l'art contemporain, et dédiées à la photographie, à l'art vidéo, à l'image, le Musée du Jeu de Paume à Paris, fondé en 2006, accueille les machinimas depuis 2009 mais uniquement dans sa programmation satellite; de même, la scène conventionnée pour les «Écritures Numériques » d'Enghien, créée en 2007, accueille ceux-ci mais surtout en marge des principaux festivals. C'est sous l'initiative d'écoles de beaux-arts que les machinimas font sur la période l'objet de récompenses et de prix. Le studio national des arts contemporains du Fresnoy expose depuis 2008 un panorama de machinimas ; le festival international Gamerz, existant depuis 2004, créé par l'École d'Art d'Aix-en-Provence et subventionné par la ville, expose et récompense des machinimas depuis 2009 , en nommant une curatrice.

Enfin, des milieux très localisés du monde du cinéma organisent sur la même période des rapprochements avec cette nouvelle écriture venue du numérique. C'est l'espace du « cinéma expérimental » qui a donné le plus d'écho à ces productions nouvelles : en France, le festival Hors Pistes, créé en 2004, leur consacre une place depuis 2008 ; de même, les festivals internationaux de Hyères et de Knocke-le-Zoute, dévolus au cinéma expérimental, ont accueilli et parfois primé des machinimas. Mais les machinimas sont aussi très présents dans les festivals de court-métrage, comme en France dans celui de ClermontFerrand depuis 2007, ou surtout celui de Belfort qui en a primé un en 2011. Ils ont enfin commencé à apparaître en 2010 dans les festivals de film d'animation, comme celui d'Annecy.

La plupart des auteurs qui obtiennent des prix sont des artistes diplômés des écoles de cinéma ou des écoles de beaux-arts. L'exemple français l'illustre assez bien : une consécration exceptionnelle est accordée à un groupe de cinq auteurs qui cumulent de nombreux prix et qui viennent d'écoles de beaux-arts et de cinéma. Avec Hôtel, par exemple, une œuvre de 60 minutes produite en 2009, Benjamin Nuel, un artiste issu de l'école des Arts Décoratifs de Strasbourg et de l'École des Beaux-Arts du Fresnoy à Tourcoing, fut 
lauréat dans différentes catégories d'instances de consécration (festival Atopic, festival de cinéma expérimental). Né en 1981 à Saint-Étienne, Benjamin Nuel avait d'abord réalisé des formats plus courts ( La fin du monde en 2006 (11 minutes), Pattern Island en 2008 (6 minutes). Il bénéficie en 2008 d'aide à la maquette du DICREAM pour faire ce film, complétée par une aide de la DRAC. Suite au succès critique remporté par Hôtel, il bénéficiera aussi d'une aide complémentaire venant du CNC. Avec Trashmaster, un long-métrage de 88 minutes, Mathieu Wechsler est lui aussi lauréat dans différentes catégories d'instances de consécration. Il a une licence de cinéma; n'ayant pas trouvé de financement ou d'avance ou d'aide pour faire ses premiers films, il décide de se tourner pour des raisons « alimentaires » vers la publicité ; parallèlement, il fait le choix de passer par les moteurs de jeux vidéo pour produire, sur son temps « libéré », un long-métrage. Il passe un an à réaliser son film tout seul chez lui, déclarant y passer « environ 1000 heures de travail».

\section{Une esthétique de la pauvreté}

Ce qui fait que les machinimas deviennent des œuvres d'auteur, c'est inversement qu'ils reposent sur l'assomption d'une " pauvreté » esthétique. Les supports expressifs sont pauvres. Ainsi, la synchronisation labiale est hors de portée, ce qui conduit les auteurs à privilégier la « voix off » ou à privilégier des costumes pour les personnages qui font qu'on ne voit pas bien leur visage : par exemple, les premières séries de machinimas faites sur Halo l'ont été précisément en choisissant que les personnages aient des casques, parce qu'il s'agissait d'éviter qu'on voie leurs bouches mal synchronisées et leurs visages; dans le long-métrage Trashmaster, l'œuvre est construite avec une voix off dans toute la première moitié, en réaction à l'impassibilité faciale des avatars.

La pauvreté du jeu des visages et de l'acteur est paradoxalement un moyen de faire émerger une légitimité esthétique. Cela sur un registre très différent du cinéma d'auteur de la Nouvelle Vague, qui a construit une onto-théologie du visage de l'acteur. Alors que le cinéma d'auteur, et la « doxa auteuriste » (Mary, 2007, p. 58) dans ses constructions fondatrices, était fondé sur l'accomplissement phénoménologique du jeu des «modèles », sur une « onto-théologie du visage de l'acteur » (l'expression est de Bazin, lorsqu'il parle de Robert Bresson), cette capacité, à travers un visage, à constituer des images-temps (pour citer Deleuze, souvent invoqué par ces auteurs). Le film d'auteur au cinéma était le moyen de voir émerger une "permanence d'être 》, la "trace la plus lisible de l'âme », le «masque d'un destin spirituel » (expressions prélevées par Mary dans le corpus de critiques du cinéma d'auteur). Dans le cas 
des machinimas, ce mouvement est contrarié par la pauvreté des musculatures et des possibilités de personnalisation des « marionnettes virtuelles » utilisées. L'art des machinimastes, pour les tenants de leur légitimation esthétique, s'apparente ainsi plutôt à la tradition du théâtre d'effigie (Nitsche, 2005), avec son expressionnisme rugueux et les mouvements simplifiés de ses poupées, comiques à force d'être mécaniques, qu'à l'art cinématographique.

C'est principalement autour de cette « esthétique de la " pauvreté », qui peut être comparée à la « basse définition » dont Nicolas Thély a montré l'importance dans la consécration artistique des films faits à la webcam, que se structure la rencontre des machinimas et de l'art numérique. Les moteurs en temps réel (par opposition aux moteurs pré-calculés) proposent une variété de personnages et de décors, mais pâtissent cruellement d'une latitude expressive extrêmement sommaire. Ce défaut est alors sublimé en atout. La mécanique grotesque des mouvements, l'imbécillité attendrissante des sourires réduisent toute ambition d'intimité et condamnent à l'action (le navrant Time travelers, machinima de Boyd, Amsterdam et Canton), au décoratif (Red zone ou Birth, machinima de Iono Allen) ou à la vanité immature à observer sa vie virtuelle (A Year in the life, machinima de Boyd et Amsterdam).

\section{Un profil émergent d'artistes « natifs du numérique » inscrits dans un réseau de diffusion alternatif}

Il est caractéristique que, à côté de cette trajectoire, émerge un profil d'auteurs obtenant une forte audience sur les plates-formes vidéo numériques, et ne faisant presque l'objet d'aucune consécration, si ce n'est exclusivement sous la forme de prix du public ou du jury dans les instances culturelles. Ces auteurs voient se privilégier pour eux des carrières en tant que professionnels de la publicité ou de la télévision. Dans certains cas, leur choix de privilégier la distribution sur les plates-formes de vidéo du numérique les prive de possibilité de concourir dans les festivals, ces derniers réclamant, pour leur programmation, une exclusivité sur la diffusion. En revanche, leur forte notoriété sur les platesformes du numérique leur permet d'accéder à des revenus complémentaires.

Nous illustrons cette dernière trajectoire par un profil caractéristique. Michael Borras, né en 1983, fabrique des machinimas depuis 2000. Autodidacte, il se décrit comme " artiste du multimédia et des réseaux », et a multiplié l'invention de formes sociales lui permettant d'inscrire sa trajectoire dans des dyna- 
miques d'appartenance à des collectifs : il se décrit comme le fondateur du mouvement « French Trash Touch » (qui commence à être attesté vers 2003), comme membre de l'internationale, qu'il a fondée, dite " échantillonniste ", et comme l'initiateur du «Spamm », le « musée des Arts super-modernes ». Il fait des machinimas depuis 2007, date à laquelle il a lancé une websérie selon cette méthode. Il est un acteur important de ce qu'on pourrait appeler un réseau artistique « alternatif » dont la plupart des auteurs sont concentrés géographiquement en région parisienne, et focalisé autour de l'art numérique, de l'art en réseau, de productions virales à très forte notoriété sur les plates-formes de distribution vidéo. Sous le pseudonyme de Systaime, il réalise régulièrement de nombreuses œuvres en ligne, vidéos, remix, webfilms expérimentaux, clips, ainsi que des installations et des performances, disant vouloir " pousser au maximum les limites de cet objet qu'on nous met entre les mains ». Malgré cette relative absence de consécration dans les institutions d'art, il est fortement inséré dans une carrière professionnelle sous plusieurs activités et métiers : il est DJ, il a fait la première partie de concerts de vedettes (Charlélie Couture) au Bataclan, il a réalisé une série de clips diffusés sur le site internet du journal Libération, à tonalité d'humour politique. Cette forte activité lui permit d'ailleurs, en 2011, d'être invité ponctuellement dans des institutions comme la villa d'Arson sur Nice. Frédéric Nakache, quant à lui, né en 1972, est un artiste autodidacte qui a été repéré par des galeristes et des musées d'art contemporain. Son parcours de vidéaste s'est effectué sans formation spécialisée dans cette pratique artistique.

Les auteurs de machinimas qui visent plus que le simple profil de « l'amateur enrichi » essuient donc une réception mitigée : ils reçoivent une réaction conservatrice des métiers de l'animation professionnelle, les instances de consécration auxquelles ils sont soumis légitiment de manière caractéristique des auteurs de vidéos ayant un background Beaux-Arts/Cinéma.

\section{CONCLUSION}

Les spectateurs les plus jeunes ont pris l'habitude d'être de plus en plus actifs vis-à-vis des univers qu'on leur propose. Ils adorent partager, commenter, analyser ou parodier telle ou telle séquence. Le succès des machinimas ou des séquences amateurs auprès de ces publics, répond ainsi à ce besoin qu'ont les joueurs d'être explorateurs, expressifs et ironiques. Par ces séquences audiovisuelles faites avec les moteurs des jeux vidéos, ils donnent leurs avis sur le jeu, déconstruisent les grammaires esthétiques de leurs auteurs, en moquent 
les idéologies : bref, ils font émerger un regard critique sur cet univers, structurant autour de ces micro-créations une transformation de l'industrie culturelle en « champ esthétique».

Les lieux numériques d'échange de ces détournements et regards critiques sont les nouveaux sièges des dynamiques d'apprentissage de la création. Leur essor est un enjeu central de la numérisation. Dans l'industrie du jeu vidéo, ces communautés en ligne permettent de compenser la faiblesse des circuits de reconnaissance patrimoniale. Les machinimas, qui ont ici été étudiés dans le détail, constituent ainsi, pour les passionnés qui se destinent à un des métiers de la création dans le secteur, à la fois un moyen d'apprendre les codes culturels des différents genres, et une manière de se former à la grammaire cinématographique qu'il est nécessaire de maîtriser pour fabriquer un « bon produit ». Paradoxalement, ce double rôle « buissonnier» des communautés de partage de machinimas s'est récemment accru depuis 2005 avec l'apparition d'une spécialisation professionnelle sur les formations et la généralisation sur tout le territoire d'écoles pour les métiers du jeu vidéo. Il serait intéressant d'étudier la place que tiennent les «machinimas » chez les jeunes qui suivent ces parcours professionnels spécialisés. En tout état de cause, les univers de partage deviennent un lieu illustratif de la transformation des publics de l'art en spectateurs discutants et actifs, une sorte de Fun Palace selon la métaphore utilisée par Jean-Louis Fabiani en référence à l'architecture d' « antibâtiment » imaginée par Cédric Price. Ils sont emblématiques de nouveaux objets culturels qui indiquent une altération du modèle classique de la légitimité culturelle, l'émergence de processus endogène de production de la valeur artistique à partir de la discussion sur les œuvres à l'intérieur des genres, et le caractère composite de publics qui se situent au-delà de l'anxiété à propos des questions de légitimité. 


\section{RÉFÉRENCES}

AARSETH, E., 2001, « Computer Game Studies, Year One », Game Studies 1 (1) AUGROS, 1996, L'argent d'Hollywood, Paris, L'Harmattan.

AURAY N. \& DAVIDOVICI-NORA, M., 2011, « Caractéristiques technico-socioéconomiques et principales tendances d'évolution de l'industrie des jeux vidéo dans le monde ", dans Synthèse des filières des industries culturelles, document rédigé dans le cadre du projet PANIC, projet soutenu par l'ANR, 2010, p. 94-160.

BALZERANI, M., 2009, « Journal intime d'une critique d'art à l'ère du Web 2.0 », Le Magazine électronique dpi.studioxx, septembre (http://dpi.studioxx.org).

BEAU, F., 2006, « L'âge du craftware », Culture d'univers, p. 269-282, Fyp, Limoges.

BEUSCART, J.-S. et al., 2009, « Pourquoi partager mes photos de vacances avec des inconnus ? Les usages de Flickr », Réseaux, 2009/2, n 154, p. 91-129.

BEUSCART J.-S., CREPEL, M. « Les trajectoires de notoriété sur le Web 2.0 : quatre figures de l'engagement dans la pratique artistique en ligne », Communication au Colloque Digital Life Lab: « La participation des amateurs dans l'univers numérique », Paris, 18 mars 2011.

BISKIND, P., 2002, Le nouvel Hollywood, Paris, Le Cherche Midi [traduction de Easy Riders, Raging Bulls, Simon and Schuster].

BLANCHET, A., 2010, Des pixels à Hollywood, Paris, Pix’n Love Éditions.

BLANCHET, A., 2011, «Adaptation des films de cinéma en jeu vidéo : une analyse statistique », Ina Global [accessible en ligne : http://www.inaglobal.fr/jeu-video/article/adaptation-des-films-de-cinema-en-jeux-video-une-analyse-statistique?tq=8].

BOLTER, J., GRUSIN, R., 1999, Remediation. Understanding New Media, MIT Press.

BOURGATTE, M., THABOUREY, V., dir., 2012, Le cinéma à l’heure du numérique. Pratiques et publics, Mkf éditions.

BRUNO, P., 1993, Les jeux vidéo, Syros La Découverte, Paris.

CARDON, D., 2005, « Les innovations ascendantes », Internet Actu, juin.

CARDON, D., ROTH C. et FOUETILLOU, G. « Trajectoire de consécration des amateurs dans le monde numérique », Communication au Colloque Digital Life Lab: « La participation des amateurs dans l'univers numérique », Paris, 18 mars 2011.

CERTEAU, M. de, 1980, L'Invention du Quotidien. Vol. 1, Arts de faire. Paris, Union générale d'éditions 10-18.

COSTIKYAN, G., 2002, « I Have No Words \& I Must Design: Toward a Critical Vocabulary for Games », in MÄYRÄ, ed., CGDC Conference Proceedings, Tampere University Press, p. 9-34. 
DUJARIER, M.-A., 2010, Le travail du consommateur, Paris, La Découverte.

FABIANI, J.-L., 1993, « Sur quelques progrès récents de la sociologie des œuvres », Genèses, $\mathrm{n}^{\circ} 11$, p. 148-167.

FABIANI, J.-L., 2008, Après la culture légitime. Objets, publics, autorités. Paris, L'Harmattan.

FLICHY P., 2004, « L'individualisme connecté entre la technique numérique et la société », Réseaux, vol. 22, n 124.

FLICHY P., 2010, Le sacre de l'amateur : sociologie des passions ordinaires à l'ère numérique, Paris, Seuil.

GENVO S., 2009, Le jeu à son ère numérique : comprendre et analyser les jeux vidéos, Paris L'Harmattan.

GUARDIOLA, E., 2005, «L'histoire que nous faisons vivre aux joueurs : la structure ludo-narrative ", in S. Genvo, ed., Le game design de jeux vidéo. Approches de l'expression vidéoludique, Paris, L'Harmattan, p. 161-174.

GUNTHERT, A., 2008, « L'empreinte digitale. Théorie et pratique de la photographie à l'ère numérique ", in Careri, G., Rüdiger, B. (eds.), Face au réel. Éthique de la forme dans l'art contemporain, Paris, Archibooks, p. 85-95.

HÄFLIGER, S., JÄGER, P., von KROGH, G. Under the Radar, Industry entry by user entrepreneurs, Research Policy.

HÄFLIGER, S., REICHEN, Ph., JÄGER, P., von KROGH, G., 2009, « Modding as rating behavior in virtual communities: The case of Rooster Teeth Productions ", Online communities ans social computing, volume 5621, Springer Berlin Heidelberg, p. 197-206.

HENNION A., 2007, La Passion musicale. Une sociologie de la médiation, Paris, Métailié.

JENKINS H., 2006, Fans Bloggers and Gamers: Media Consumers in a Digital Age, New York, New York University Press.

JONES R., 2008, Machinimateur Wanted: The Professionalization of Machinima, http:// clients.jordanjennings.com/Mediascape/Fall08/Spring08_MachinimateurWanted.pdf.

KING, G., 2002, New Hollywood Cinema, An Introduction, New York, Columbia.

KROGH G. von, HÄLFIGER, S., JÄGER, P., 2010, « Under the radar: Industry Entry by user entrepreneurs », Research Policy, 39, p. 1198-1213.

LOWOOD, H., NITSCHE, M., 2011, The Machinima Reader, MIT Press.

LOWOOD H., 2004, « Playing History with Games steps towards historical archives of computer gaming », Presented at the Electronic Media Group Annual Meeting of the American Institute for Conservation of Historic and Artistic Works Portland, Oregon June 14. 
LOWOOD H., 2006, « High-Performance Play: The Making of Machinima », Journal of Media Practice, 7:1, p. 25-42.

MAIGRET, E., 2000, « Les trois héritages de Michel de Certeau. Un projet éclaté d'analyse de la modernité », Annales, 3, p. 511-549.

MANOVITCH, L., 2007, The Language of New Media, MIT Press.

MANOVITCH, L., in H. Lowood, M. Nitsche, eds., The Machinima Reader, MIT Press.

MARINO P., 2007, «3D game-based filmmaking: The art of machinima », Scottsdale, AZ: Paraglyph, Clarke Andy \& Mitchell Grethe (eds.), Videogames and Art: Intersections and Interactions, London, Intellect, p. 59-79.

MARY, P., 2006, La Nouvelle vague et le cinéma d'auteur. Socio-analyse d'une révolution artistique, Paris, Seuil.

MURRAY, J. H., 1997, Hamlet on the Holodeck: The Future of Narrative in Cyberspace, New York: Free Press.

NITSCHE, 2005, « Film live: And Excursion into Machinima », in Developing Interactive Narrative Content: sagas_sagasnet_reader, ed. by Brunhild Bushoff, Munich, High Text, p. 210-243.

PEARCE Celia, 2003, « L'émergence de la co-éditique, prochaine révolution interactive », in H. Fisher (ed.), Les défis du cybermonde, Laval (Canada), p. 173-183.

PICARD M., 2006, Machinima: Video Game As An Art Form?, CGSA 2006 Symposium.

SIHVONEN T., 2009, Players Unleashed! Modding The Sims and the Culture of Gaming, Electronic book text, Silverstone.

SOTAMAA, O., 2007, "Let Me Take You to The Movies", Convergence. The International Journal of Research into New Media Technologies, vol. 13 (4), 383-401.

STIEGLER, B., 2011, Ce qui fait que la vie vaut la peine d'être vécue. De la pharmacologie, Paris, Flammarion.

TAYLOR, T., 2006, Play Between Worlds, Cambridge, MIT Press.

VOIROL, O., 2011, « Retour sur l'industrie culturelle », 2011/2, n 166, p. 125-157.

von HIPPEL, E., 2005, Democratizing Innovation, MIT Press. 